\title{
New Concept of Plasma-Forming : PLASMA Physics for Your Skin Treatment
}

\author{
Changwon Choi, MEng \\ SHENB Medical Inc., Seoul, Korea
}

Received March 24, 2020

Revised April 8, 2020

Accepted April 14, 2020

\section{Correspondence \\ Changwon Choi \\ SHENB Medical Inc., Seoul 04796, Korea \\ E-mail: lab3@shenb.com \\ https://orcid.org/0000-0002-0976-9444}

(C) Korean Society for Laser, Dermatology and Trichology

(c) This is an open access article distributed under the terms of the Creative Commons Attribution NonCommercial License (http://creativecommons.org/ licenses/by-nc/4.0) which permits unrestricted noncommercial use, distribution, and reproduction in any medium, provided the original work is properly cited.
The elements that form the Earth appear in the form of solids, liquids, and gases, transitioning between states thereof with changes in temperature. When there is more energy applied to these three basic state, it becomes the plasma. A plasma is formed when there are equal amounts of ionized particles and electrons, called a quasi-neutral state. Plasma with various properties is used in various applied sciences, industries, and medical fields. When artificially generated plasma is used for industrial or medical use, it can be divided into high temperature or thermal plasma and cold plasma according to the temperature. High-temperature plasma is mainly used for industrial purposes such as nuclear fusion, incineration, and metal cutting, whereas low-temperature plasma is used for light-emitting sources, medical fields, and equipment. The most typical characteristic of plasma is the radicals that are generated in various ways. the radicals generated by plasmas are thought to be more effective in killing cancer cells or sterilizing bacteria. Various radicals generated in the plasma destroy the cell wall of bacteria and have a strong sterilizing effect and interestingly, living tissues are hardly damaged under the same conditions. Therefore, plasma has a very good effect on bacterial-infected tissues, thereby can speed up the wound healing process. In particular, interest in plasma for dermatological treatment is increasing, and cases of use for wound healing have been reported in the UK and Germany. In addition, recently, cases of use in acne, athlete's foot, freckles, and atopy have been continuously reported, and as interest in plasma has increased, efforts to apply it to various skin treatments are increasing. Plasma will continue to be actively researched in more fields in the future. Through such efforts, it will be necessary to develop purpose-built plasma generating devices that are easier and more convenient to use. Furthermore, additional research is needed to determine how unstable plasma energy can be accurately adjusted and to develop quantitative methods with which to measure radicals present in a plasma.

Key words

Plasma, Radicals, Nitrogen, Argon

Supplementary video fille: This article contains supplementary material (It is available at https://youtu.be/3Kv8bH7R13M). 


\section{INTRODUCTION}

The elements that form the Earth appear in the form of solids, liquids, and gases, transitioning between states thereof with changes in temperature. For example, water becomes a solid (ice) at low temperatures, a liquid as the temperature rises, and a gas (steam) at even higher temperatures.

What happens when more energy is applied to these three basic states?

If the energy applied is greater than the energy binding two molecules, they are separated into atoms, and if even more energy is applied, electrons in the outermost part of the atom are separated from the atom. This process is called ionization [1].

In an ionized state, a plasma is formed when there are equal amounts of ionized particles and electrons, called a quasi-neutral state. In other words, a plasma is a gas in an ionized state in which the number of ions equals that of electrons.

Plasma was first discovered in 1928 by Irving Langumuir, an American physicist employed by General Electric, while studying gas discharge.

At a macro perspective, plasma is electrically neutral. Looking deeper, however, one can see that, unlike gases wherein particles with opposite charges (electrons and protons) are mixed together, ions and electrons of different charges are separated, generating a current and magnetic field due to the flow of charges. Plasma is also a chemically reactive medium.

Plasmas have unusual physical and chemical properties. First, because plasma is formed at high temperatures, the particles that make up the plasma are high in kinetic energy. Second, plasmas have high conductivity and allow electricity to pass through them, like metal. Third, they can be chemically excited to increase reactivity. Due to these properties, plasmas can also be used to generate light.

How is that plasmas have these properties?

These properties arise as a results of collisions between electrons and gas molecules. When an electron collides with a gas molecule with very little energy, it will bounce off, with no change in kinetic energy. However, if the collision occurs with large enough energy (e.g., due to high temperatures or in a strong magnetic field), the electron will transition to a higher orbital energy level: molecules in this higher energy state are called excited molecules. In a very short amount of time, electrons at a higher orbital level in this excited state will return to their normal state, and the remaining energy will give off an illumination effect. Meanwhile, if the energy of the collision is higher than the orbital binding energy for the electron, the electron escapes and ionization occurs. At high enough collision energy, the bonds that hold molecules can be broken, generating molecules called chemically active species or radicals. These radicals generally return to a stable state in a short amount of time, chemically recombining with other nearby radicals.

Plasmas are used in various field of applied science, industry, and medicine and can be divided into thermal and cold plasmas depending on the temperature at which they are generated [2]. Thermal plasmas are produced at temperatures reaching thousands to tens of thousands of degrees Celsius and are used for various purposes as highly efficient heat sources: for example, nuclear fusion, incineration, metal cutting, and non-degradable gas treatment. Conversely, cold plasmas are produced at temperatures in the tens to hundreds of degrees Celsius using primarily radiofrequency or microwave devices. Although the temperatures of these plasmas are relatively low, the temperatures of the electrons that form the plasma are high, causing corona and arc discharges. These plasmas are used in semiconductor manufacturing, light emission sources, and others.

Various joint studies in the Netherlands, United States, Germany, and United Kingdom have helped usher in the use of cold plasmas in the biomedical field. In the 2000s, Eva Stoffels in the Netherlands reported that had plasma had a killing effect on various mammalian cells and microorganisms, including cancer cells. Since 2005, remarkable advances have resulted in the use of plasmas for sterilization and to stimulate cancer cell death, hemostasis, and wound healing.

\section{CONCLUSION}

While plasma itself does show some effects on cells, the radicals generated by plasmas are thought to be more effective in killing cancer cells or sterilizing bacteria [3-5]. The radicals generated by plasma have been found to destroy the cell wall of bacteria while preserving living tissue, thereby speeding up the wound healing process. The hydroxyl radicals, ions, electrons, photons, ionized gas, and very small amount of ultraviolet light emitted from plasmas are harmless to the human body. Moreover, nitric oxide radicals produced when plasma is generated using nitrogen gas has been found to promote cell differentiation and wound healing [6-10]. In animal models, researchers have found that these radicals are effective against tissue infections and acute and chronic inflammation. This makes plasmas potentially good options with which to address problems with resistance to existing antibiotics. 
Numerous reports have also described the use of plasma in cancer treatment and other medical purposes. Research has indicated that plasmas are particularly effective at killing leukemia and melanoma cells, in addition to others. Plasma-generated radicals have also been shown to be important to blood coagulation [11,12]. Additionally, with an increasing number of studies reporting cases of acne, tinea pedis, melasma, and atopic dermatitis being successfully treated with plasma, interest in the use of plasma for skin treatments has grown tremendously [13-16].

In the medical field, cold plasmas are used to prevent unwanted thermal reactions and unintended damage stemming from overheating. However, the necessity of a vacuum chamber to produce a plasma has acted as a barrier to expanding the range of applications of these plasmas [17]. Meanwhile, studies dating back to the 1980s have shown that plasmas can be generated at atmospheric pressure. At high atmospheric pressure, a greater discharge voltage is required to generate plasma, compared to that required at low pressure in a vacuum. According to Paschen's law, at a constant distance between electrodes, the mean free path between electrons is shortened as pressure increases, resulting in lower collision energy, which makes it difficult to discharge electrons to form a plasma at high pressure. To

Table 1. Value of ionization energy at atmospheric pressure of several elements

\begin{tabular}{ccc}
\hline \multirow{2}{*}{ GAS } & \multicolumn{2}{c}{ Breakdown Voltage at Atmospheric Pressure } \\
\cline { 2 - 3 } & $\mathrm{AC}\left(\mathrm{kV}_{\mathrm{RMS}} / \mathrm{cm}\right)$ & $\mathrm{DC}(\mathrm{kV} / \mathrm{cm})$ \\
\hline Air & 23 & 33 \\
$\mathrm{He}$ & 1.3 & 1.63 \\
$\mathrm{Ar}$ & 3.4 & 4.8 \\
$\mathrm{~N}_{2}$ & 22.8 & 32 \\
\hline
\end{tabular}

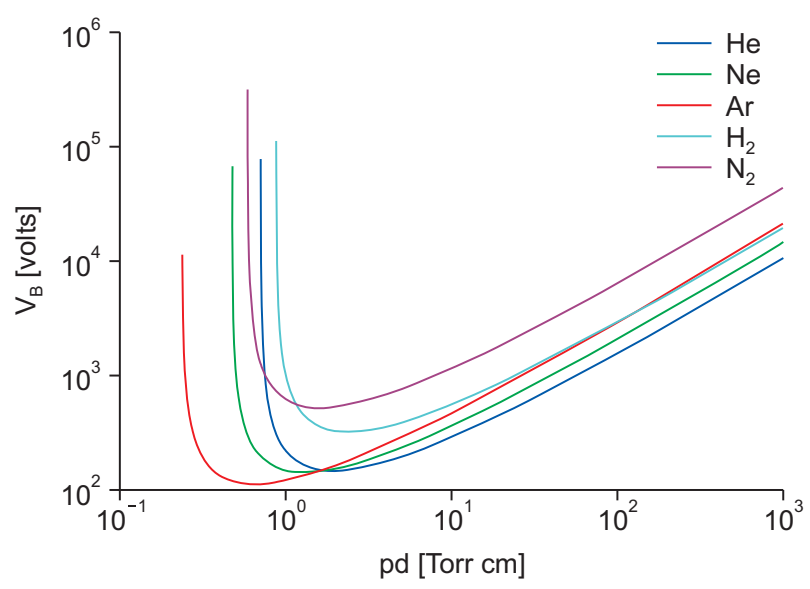

Fig. 1. Paschen's curves. solve this, the voltage must be increased (Table 1, Fig. 1).

Plasma can be generated at various frequencies, although the use of a high power and microwave frequencies at a few $\mathrm{GHz}$ close to the resonant frequencies of plasma electrons is more advantageous. With microwave plasma devices, it is relatively easy to discharge gases, such as nitrogen, for which generating a plasma is difficult, by applying energy to atoms using microwaves in the $2.45 \mathrm{GHz}$ band. Accordingly, using microwaves has the advantage of allowing researchers to generate plasmas with various types of gases.

For medical purposes, current plasma devices utilize the ionization of various inert gases, such as helium, argon, nitrogen, and neon. These gases can be used to generate plasmas at both low and atmospheric pressure. Medical devices using inert gases as a medium primarily produce plasmas in the form of a plume.

In plasmas, radicals separate and re-combine in a very short amount of time, forming a "plasma cocktail," each

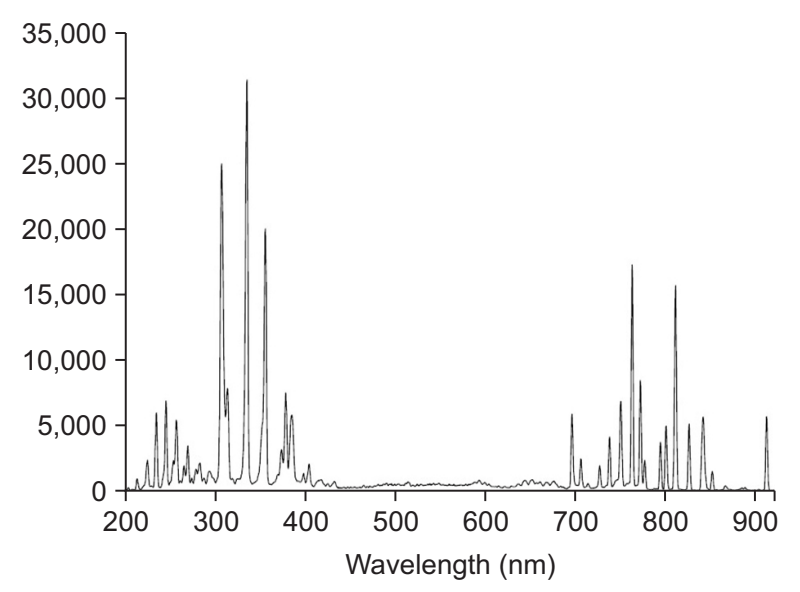

Fig. 2. Light emission spectra of nitrogen and argon.

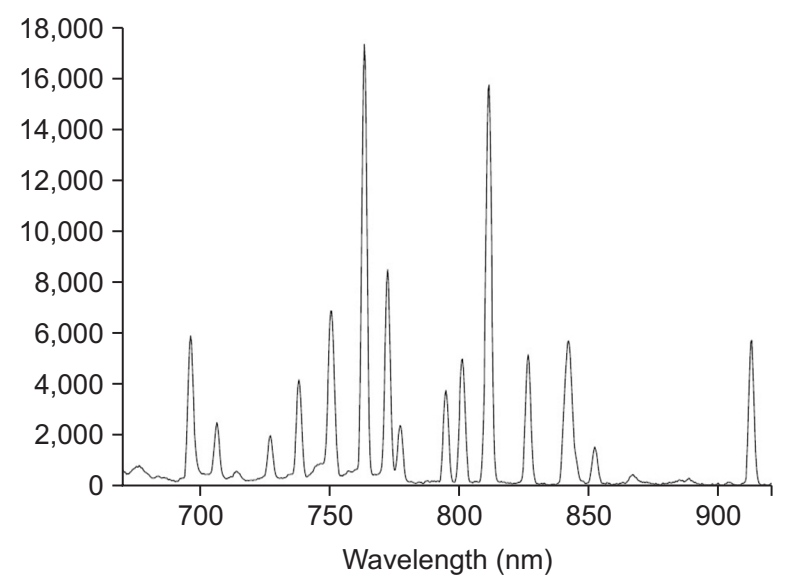

Fig. 3. Light emission spectra of argon plasma. 


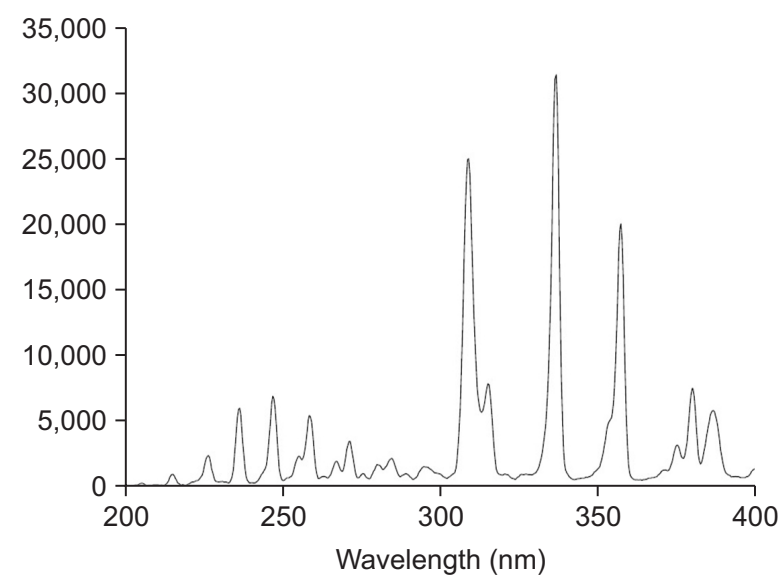

Fig. 4. Light emission spectra of nitrogen plasma.

with different effects on cells. In order to verify the presence of radicals in plasmas generated by atmospheric pressure devices, researchers analyze the light emission spectra of plasma plumes. The spectrum generated by nitrogen ranges from $300 \mathrm{~nm}$ to $400 \mathrm{~nm}$, and the light emitted by ionized nitrogen molecules can be clearly seen at $337 \mathrm{~nm}$ and $358 \mathrm{~nm}$. That for argon spans from 600 to $900 \mathrm{~nm}$, with light emitted by the discharge of electrons primarily visible at $696 \mathrm{~nm}, 763 \mathrm{~nm}$, and $810 \mathrm{~nm}$ (Fig. 2-4) [18,19].

To verify the occurrence of nitric oxide and hydroxyl radicals in these light emission spectra, researchers can refer to the previously published paper entitled "Effects of cold atmospheric plasma generated in deionized water in cell cancer therapy" by Zhitong Chen, Li Lin, Xiaoqian Cheng, Eda Gjika, Michael Keidar. In accordance with their results, nitric oxide radicals can be observed by light emitted between 250 to $300 \mathrm{~nm}$ and hydroxyl radicals can be verified by light emitted at $309 \mathrm{~nm}$ (Fig. 5).

\section{DISCUSSION}

Plasma will continue to be actively researched in more fields in the future. Through such efforts, it will be necessary to develop purpose-built plasma generating devices that are easier and more convenient to use. Furthermore, additional research is needed to determine how unstable plasma energy can be accurately adjusted and to develop quantitative methods with which to measure radicals present in a plasma.

\section{CONFLICT OF INTEREST}

No potential conflict of interest relevant to this article was reported.

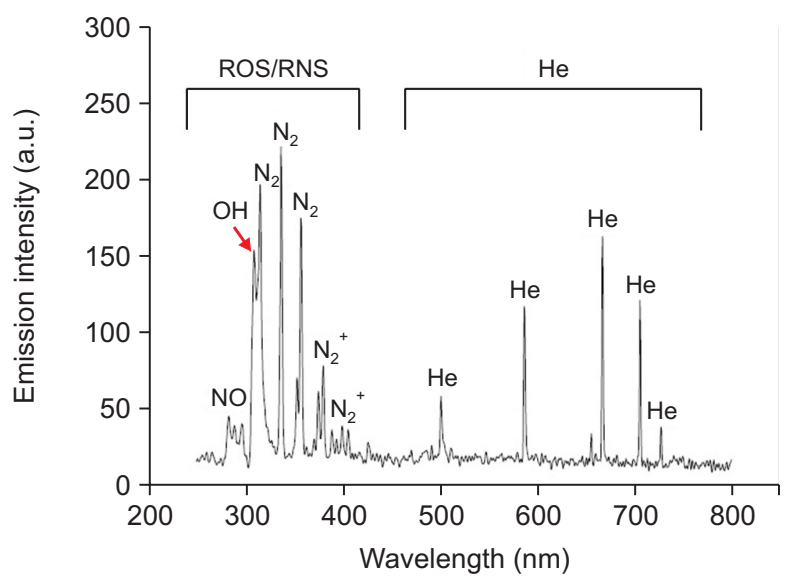

Fig. 5. Confirmation of occurrence of $\mathrm{OH}$ and $\mathrm{NO}$ radicals depends on a plasma generating.

\section{REFERENCES}

1. von Woedtke T, Schmidt A, Bekeschus S, Wende K. Introduction to plasma medicine. In: Metelmann HR, von Woedtke T, Weltmann KD, editors. Comprehensive Clinical Plasma Medicine. Cham: Springer; 2018. p.3-21.

2. Helmke A, Gerling T, Weltmann KD. Plasma sources for biomedical applications. In: Metelmann HR, von Woedtke T, Weltmann KD, editors. Comprehensive Clinical Plasma Medicine. Cham: Springer; 2018. p.23-41.

3. Canady J, Gordon S, Zhuang T, Wigh S, Rowe W, Shashurin A, et al. Cold Atmospheric Plasma (CAP) combined with chemoradiation and cytoreductive surgery: the first clinical experience for stage IV metastatic colon cancer. In: Metelmann HR, von Woedtke T, Weltmann KD, editors. Comprehensive Clinical Plasma Medicine. Cham: Springer; 2018. p.163-83.

4. Seebauer C, Metelmann HR, Witzke K, Pouvesle JM. Palliative treatment of head and neck cancer. In: Metelmann HR, von Woedtke T, Weltmann KD, editors. Comprehensive Clinical Plasma Medicine. Cham: Springer; 2018. p.185-95.

5. Bekeschus S, Pouvesle JM, Fridman A, Miller V. Cancer immunology. In: Metelmann HR, von Woedtke T, Weltmann KD, editors. Comprehensive Clinical Plasma Medicine. Cham: Springer; 2018. p.409-19.

6. Graves DB, Bauer G. Key roles of reactive oxygen and nitrogen species. In: Metelmann HR, von Woedtke T, Weltmann KD, editors. Comprehensive Clinical Plasma Medicine. Cham: Springer; 2018. p.71-82.

7. Kim H, Kim HJ, Kim HK, Hong JY, Cho SB. Effects of argon and nitrogen plasma pulses on the skin and skin appendages in an in vivo animal model. Skin Res Technol 2020;26:81-90.

8. Preissner S, Raguse JD, Hartwig S. Treatment of split skin draft donor and recipient sites. In: Metelmann HR, von Woedtke T, Weltmann KD, editors. Comprehensive Clinical 
Plasma Medicine. Cham: Springer; 2018. p.197-200.

9. Urayama T, McGovern M. Selected settings of clinical plasma treatment. In: Metelmann HR, von Woedtke T, Weltmann $K D$, editors. Comprehensive Clinical Plasma Medicine. Cham: Springer; 2018. p.213-51.

10. Tiede R, Emmert S, Isbary G. Treatment of ulcerations and wounds. In: Metelmann HR, von Woedtke T, Weltmann KD, editors. Comprehensive Clinical Plasma Medicine. Cham: Springer; 2018. p.127-49.

11. Partecke LI, Bekeschus S, Liedtke KR. Perspectives in general surgery. In: Metelmann HR, von Woedtke T, Weltmann KD, editors. Comprehensive Clinical Plasma Medicine. Cham: Springer; 2018. p.347-54.

12. Hilker L, von Woedtke T, Titze R, Weltmann KD, Motz W, Wollert HG. The use of Cold Atmospheric Pressure Plasma (CAP) in cardiac surgery. In: Metelmann HR, von Woedtke T, Weltmann KD, editors. Comprehensive Clinical Plasma Medicine. Cham: Springer; 2018. p.201-211.

13. Cho SB, Kim HJ, Kim H, Yoo KH. Argon plasma: a new approach for the effective treatment of inflammatory acne vulgaris and enlarged pores in Asian patients. Med Laser 2018;7:97-101.
14. Kim H, Kim HJ, Cho SB. Therapeutic effect of low-energy nitrogen plasma pulses on tinea pedis. Med Laser 2019;8:2831.

15. Kim HJ, Kim H, Kim YK, Cho SB. Treatment of refractory melasma with microwave-generated, atmospheric-pressure, non-thermal nitrogen plasma. Med Laser 2019;8:74-9.

16. Adhikari M, Ali A, Kaushik NK, Choi EH. Perspective in pigmentation disorders. In: Metelmann HR, von Woedtke T, Weltmann KD, editors. Comprehensive Clinical Plasma Medicine. Cham: Springer; 2018. p.363-400.

17. Wende K, Schmidt A, Bekeschus S. Safety aspects of nonthermal plasmas. In: Metelmann HR, von Woedtke T, Weltmann KD, editors. Comprehensive Clinical Plasma Medicine. Cham: Springer; 2018. p.83-109.

18. Park SJ. The study on emission spectrum characteristics of atmosphere pressure plasma. J Korean Inst Illum Electr Install Eng 2013;27:77-83.

19. Gerling T, Helmke A, Weltmann KD. Relevant plasma parameters for certification. In: Metelmann HR, von Woedtke T, Weltmann KD, editors. Comprehensive Clinical Plasma Medicine. Cham: Springer; 2018. p.43-70. 